\title{
Investigations of trapped higher order modes using a 36-cell test structure
}

\author{
T. Weiland and U. van Rienen* \\ Theorie Elektromagnetischer Felder, Technische Universität Darmstadt, Darmstadt 64289, Germany \\ P. Hülsmann, W. F. O. Müller, and H. Klein \\ Institut für Angewandte Physik, JWG-Universität Frankfurt am Main, Frankfurt am Main 60325, Germany
}

(Received 22 December 1998; published 6 April 1999)

\begin{abstract}
In future linear collider schemes, energy loss, as well as deterioration of the beam due to long-range wakefields, is a severe problem to be overcome. The wakefield effects in accelerator sections for these colliders are thought to be reduced either by damping, by detuning, or by a combination of both. While, in principle, damping is possible by selective coupling to dangerous higher order modes (HOMs), an alternative way is to prevent coherent excitation of these wakes by modifying a constant gradient accelerator tube in such a way that each following cell is progressively detuned in relation to the preceding ones ("detuned" or "stagger-tuned" structure). This should result in low transit time factors for the higher order modes. However, the disturbed longitudinal symmetry of the structure induces trapped modes especially in the lower dipole bands, which are again dangerous for the beam. The process of detuning suppresses the synchronous dipole modes with their extreme high loss factors, but it also produces many other HOMs with significant loss factors. Experimental results were compared with the predictions of MAFIA field calculations. [S1098-4402(99)00033-6]
\end{abstract}

PACS numbers: 29.17.+w, 41.75.Lx

\section{INTRODUCTION}

As an example, the $S$-band $500 \mathrm{GeV}$ Linear Collider Study (SBLC) of DESY/TUD (accelerating mode at $3 \mathrm{GHz}$ ) provides for about 5000 constant gradient acceleration structures of 180 cells with a loaded gradient of $17 \mathrm{MV} / \mathrm{m}$. It can drive bunch trains of 333 bunches each, with a spacing of $1.8 \mathrm{~m}$ from bundle to bundle. In order to achieve a high luminosity, any cumulative beam breakup along the bunch train has to be avoided. Wakefield effects driven by higher order modes (HOMs) are one of the primary sources of emittance growth. Consequently, the avoidance and suppression of these HOMs is a crucial point in all current linear collider designs [1]. In this paper, the main interest of calculations was focused on the modes of the first dipole band, since they cause the severest deflecting effects; the study of the 3rd and 6th dipole passband is required as well.

Calculations for the SBLC structure were formerly carried out with ORTHO [2] for a somewhat simplified 180cell structure with 30 landings. One of the main results revealed that not only the first $\pi$-like (similar amplitude and changing sign from cell to cell) modes influence the beam dynamics, but also about 140 other modes. A major part of these deflecting modes was shown to be trapped inside the constant gradient structure, that is, with electric and magnetic fields limited to a distinct number of cells in

*Present address: Institut für Allgemeine Elektrotechnik, Universität Rostock, Rostock 18051, Germany. the inner region of the structure and cells free of fields at the beginning and the end of the structure.

Since the phenomenon of trapped HOMs in detuned waveguides, which ought to exert a major influence on the design of damping schemes, was neither theoretically or experimentally very well known, the following HOM investigations have begun: A new test structure, proposed by Weiland, was designed with the aim of providing a structure at which $\mathrm{rf}$ measurements are easy to perform, which is easy to manufacture, which is computable with different numerical codes (MAFIA, URMEL-T, ORTHO, COM [3]) without geometric approximations, and which is expected to show appearance of trapped modes. This structure has been investigated by all the various numerical codes listed above at the Technische Universität Darmstadt. It was then constructed and built at the Institut für Angewandte Physik der JWG-Universität Frankfurt am Main, where the experiments were performed.

The design of such a structure can be accomplished by means of numerical and lumped circuit codes. While codes such as MAFIA and URMEL-T are limited to relatively short sections, codes such as ORTHO and lumped circuit codes can handle much longer sections. In order to test and determine the reliability of such codes and to investigate the behavior of trapped modes in aperiodical iris loaded waveguides, a 36-cell HOM-detuned structure was built, measured, and compared to numerical predictions. The phenomenon of modes trapped within an accelerator structure has been a crucial issue for all recent linear collider studies.

Furthermore, the incorporation of damping cells into the detuned stack of undamped cells has been considered [4]. Calculations for the damped system have been 
performed and compared with appropriate measurements (i.e., bead pull measurements and change of quality factors by the damper). In order to examine the way in which they operate, a damper cell was built and inserted into the strongly detuned 36-cell $S$-band structure

\section{DESIGN OF THE TEST STRUCTURE}

The number of cells within the test structure is limited for two reasons.

(i) For the verification of the equivalence of several numerical codes, it should be possible to calculate the whole structure with MAFIA, a well tested and reliable code. Making calculations on a very long multicell structure with MAFIA is possible only by using a two-dimensional grid, i.e., with a rotational symmetrical structure. The number of meshpoints would otherwise exceed the capabilities even of large computers. Also, the variation of cell geometry from cell to cell has to be compatible with the size of the meshes.

(ii) Another point is the following: rf measurements of long normal conducting structures are limited by the appearance of mode overlap. Therefore, a relatively short structure had to be taken in order to reduce the number of modes in every passband. The low $Q$ values cause mode overlap in passbands with low coupling factors, especially in a nonsoldered copper structure. On the other hand, the test structure had to be long enough to show trapped modes.

We decided, therefore, on the compromise to build a test structure with 36 cells (three times as long as the 12-cell structure investigated before, or one-fifth of the 180 cells of the SBLC structure).

In order to obtain a stable structure, both mechanically and thermally, a solid design was chosen. For low mechanical tolerances the geometry had to be simple. The structure was to show constant cell radius and strong linear detuning of the iris radii, with irises twice as thick as the original SBLC irises $\left(l_{i}=10 \mathrm{~mm}\right)$.

To avoid discrepancies between the experimental copper structure and the discrete mesh model used for the different numerical calculations, any curvature within the test structure was avoided and right-angled shapes were used throughout. If this had not been done, it would have been necessary to approximate the curvatures with small steps.

Because one of our main goals was to investigate trapped modes in the first dipole passband, we decided on a strong linear detuning of the iris radius from 20 to $10 \mathrm{~mm}$. For the test model we were not interested in the fundamental mode. Therefore, cell length and diameter could be chosen and kept constant for all cells $(l=23.33 \mathrm{~mm}, d=82 \mathrm{~mm})$.

Figure 1 displays the cross sectional shape of the first and last cells of the 36-cell test structure.

This structure was built from standard oxygen-free high conductivity copper. The 36 single cups were not soldered,

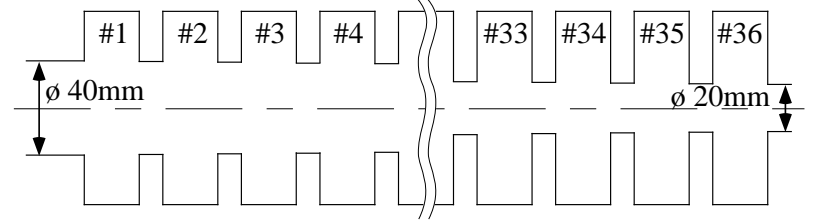

FIG. 1. Shape of the 36-cell test structure.

but rather clamped together. Each cell has four small holes in the outer wall for the insertion of tuning screws and measuring antennas. While it is sufficient for undetuned structures to have one antenna in the first cell and another one in the last cell only, for the detection of trapped modes in the detuned structure it was necessary to distribute seven antennas over the entire structure. The measuring antennas are made of semirigid cables with a coupling loop at the end, which projects into the cell only a small amount, so that its influence on the field distributions is negligible compared to the influence of production accuracy.

\section{NUMERICAL RESULTS}

Numerical calculations with the 36-cell structure were performed by MAFIA, URMEL-T, ORTHO, and COM. For the first dipole band, the most harmful one, the resonant frequencies, loss parameters, and field distributions along the structure are in quite good agreement for all four codes.

Three principally different numerical formulations were chosen for solving the field problem: (i) The codes MAFIA and URMEL-T both belong to the class of finite difference frequency domain codes. The finite integration technique [5], also belonging to this class of techniques, has been used for the discretization of Maxwell's equations. The code URMEL-T [6] solves time-harmonic eigenvalue problems for structures which are either cylindrically symmetrical or longitudinally invariant in such a way that a $2 \mathrm{D}$ discretization is sufficient to solve the field problem. In our case, the upper half of the cylindrically symmetrical structure's cross section is discretized, URMEL-T uses a triangular grid allowing for good boundary approximations with relatively coarse grids. The MAFIA [7] code consists of several solution modules which are always combined with the module $\mathrm{M}$ as preprocessor and $\mathrm{P}$ as postprocessor. Here, the $2 \mathrm{D}$ version of the time-harmonic eigenvalue module $\mathrm{E}$ was used for the solution of the field problem. In MAFIA the upper half of the structure's cross section was discretized with a Cartesian grid. Both codes have been well established and have been in worldwide use for more than 15 years. (ii) The code ORTHO is based on the mode matching technique. This technique can be applied for structures which can be split into subregions where analytical solutions of Maxwell's equations can be given as an expansion of an orthogonal series over discrete modes. The field solutions for the whole structure are then obtained by field matching at the interfaces between the subregions. This often affords more or less substantial 
simplifications of the boundary shapes. In the context of the $S$-band project, the code ORTHO was especially developed to study tapered accelerating structures. (iii) In the equivalent circuit model COM, each cell of the waveguide structure is represented by a resonant circuit. A chain of these resonant circuits serves as a model for the complete structure. The modes of the circuit are computed by MAFIA. The coupling between the cells is performed by capacitive and inductive circuit elements [8].

The first dipole passband consists of 36 modes, 23 of which are trapped (modes $4-12$ and 14-27). This is a consequence of the relatively strong detuning. Figure 2 displays the field distribution of a trapped mode calculated with MAFIA. Shown is the azimuthal component of the magnetic field near the outer wall of the cavities, which corresponds to the signal coupled by the loop antennas. The trapping is obvious: The cells \#1-\#3 (from 0.110 to $0.210 \mathrm{~m}$ ) and \#17-\#36 (from 0.643 to $1.310 \mathrm{~m}$ ) are nearly field free; the mode extends over approximately 13 cells only. The position of the 36 cells is shown by a gray bar at the top of the diagram.

These trapped modes show an increasing phase advance from cell to cell, which becomes even more evident in Fig. 3. The left end is 0-like (similar amplitude and same sign from cell to cell) and the right end is $\pi$-like with any intermediate value in between.

The loss factor or loss parameter $k$ is defined as energy loss $\Delta U$ of a charged particle crossing a resonator divided by the square of the particle charge $q$,

$$
k=\frac{\Delta U}{q^{2}} .
$$

For the loss factor calculations, the agreement between the different computer codes was not as good as that for the field distributions: the MAFIA results are shown in Fig. 4. Qualitatively, the results delivered by ORTHO and COM take the same form. However, the values from URMEL-T are somewhat scattered around that curve, being very sensitive to variations of the numerical parameters. Here numerical artifacts are suspected and, as a result,

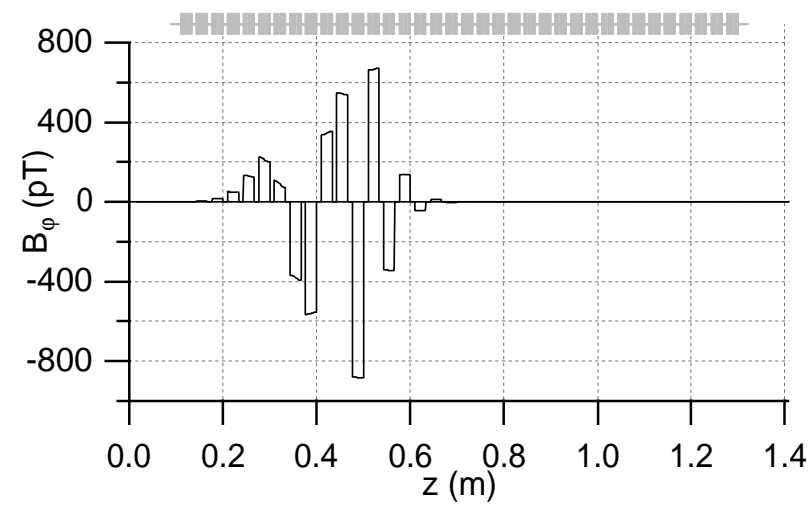

FIG. 2. Field distribution of a trapped mode from the first dipole passband $\left(f_{0}=4095.7 \mathrm{MHz}\right)$. further investigations are taking place. Mode \#13 at 4148.2 MHz (which strays out of line in Fig. 4) mainly resides in the beampipe; it is a so-called "beampipe mode," not determined by the 36 cells.

\section{METHOD OF MEASUREMENT}

The method applied is one in which the change in transmission through a cavity is measured as a bead is moved along a path inside it, the so-called nonresonant bead pull measurement $[9,10]$. The change in transmission induced by the bead, calculated with a lumped circuit representation of a resonance, is described by

$$
\Delta s_{21}=\frac{2 \sqrt{k_{1} k_{2}}}{\left(1+k_{1}+k_{2}\right)^{2}} i \Omega,
$$

with the assumption that the bead is so small that the resonant frequency $\omega_{0}$ of the cavity is changed by only a small amount. $k_{i}$ stands for the coupling factors of two ports of the resonator, $s_{21}$ denotes the transmission from port 1 to port 2 through the resonator, and $\Delta s_{21}$ is the difference in transmission for the resonator with and without the bead. The definitions used are shown here:

$$
\begin{gathered}
s_{21}=\frac{2 \sqrt{k_{1} k_{2}}}{1+k_{1}+k_{2}+i \Omega}, \\
\Omega=Q_{0}\left(\frac{\omega}{\omega_{0}}-\frac{\omega_{0}}{\omega}\right), \\
Q_{0}=\omega_{0} \frac{W}{P_{\text {loss }}} .
\end{gathered}
$$

Combining Eq. (2) with Slater's formula [10],

$$
\frac{\omega^{2}-\omega_{0}^{2}}{\omega^{2}}=-\hat{a} \frac{\left|E_{0}\right|^{2}}{W}
$$

yields

$$
\Delta s_{21}=-i \frac{2 \sqrt{k_{1} k_{2}}}{\left(1+k_{1}+k_{2}\right)^{2}} \frac{\omega_{0}}{P_{\text {loss }}} \hat{a}\left|E_{0}\right|^{2} .
$$

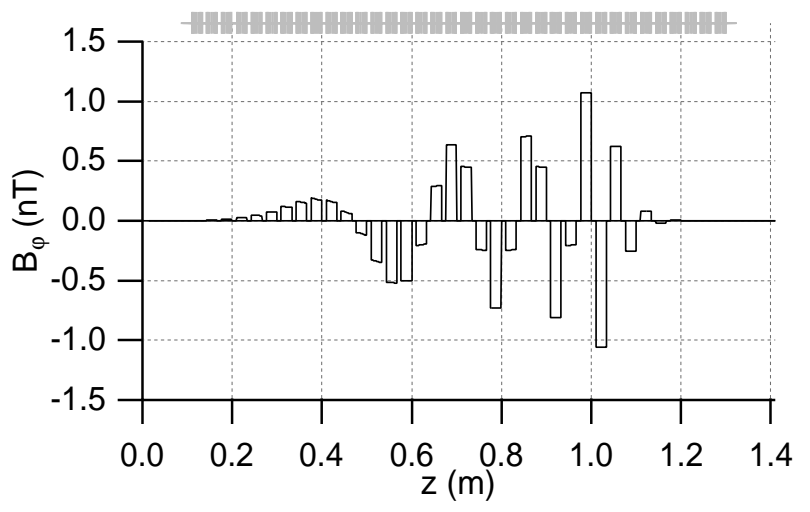

FIG. 3. Field distribution of another trapped mode from the first dipole passband $\left(f_{0}=4263.2 \mathrm{MHz}\right)$ 


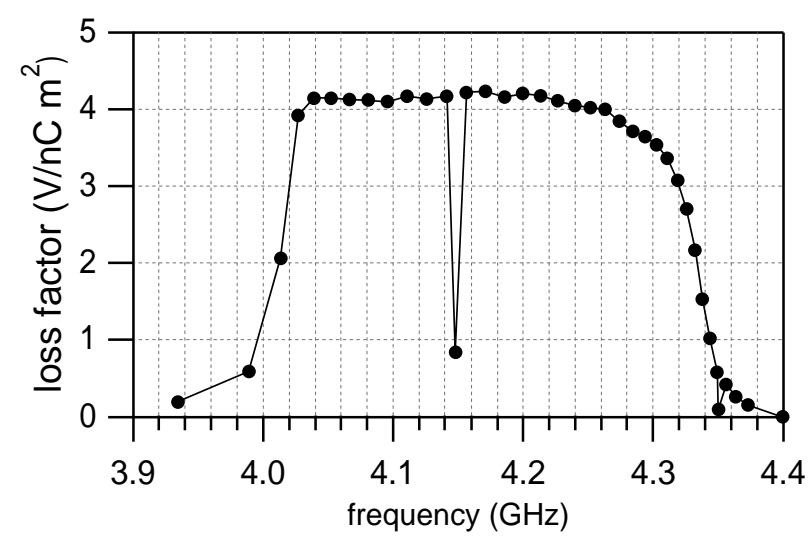

FIG. 4. Loss factor for the first dipole passband calculated with MAFIA.

We are interested in both the longitudinal and the transverse components of the electrical field. If isotropic dielectric material is used as bead and if it is shaped like a rotational ellipsoid, the factor $\hat{a}$ of the bead becomes a sum of independent quantities. The right-hand side of (7) can then be written as

$$
\hat{a}\left|E_{0}\right|^{2}=\sum_{\nu=1}^{2} a_{\nu} E_{\nu}^{2}
$$

making it possible to solve for longitudinal and transverse components separately. Two beads with different shapes (e.g., needlelike and disklike) are required.

After performing two measurements along the same pathway, the following equation is solved for $\widetilde{E}_{z}$ and $\tilde{E}_{\perp}$ :

$$
A\left(\begin{array}{c}
\frac{\Delta s_{21}^{(1)}}{a_{z}^{(1)}} \\
\frac{\Delta s_{21}^{(2)}}{a_{z}^{(2)}}
\end{array}\right)=\left[\begin{array}{ll}
1 & f_{1} \\
1 & f_{2}
\end{array}\right]\left(\begin{array}{c}
\tilde{E}_{z} \\
\tilde{E}_{\perp}
\end{array}\right),
$$

with

$$
\begin{gathered}
\tilde{E}=\frac{E^{2}}{P_{\text {loss }}}, \\
A=\frac{\left(1+k_{1}+k_{2}\right)^{2}}{2 \omega \sqrt{k_{1} k_{2}}}, \\
f=\frac{a_{\perp}}{a_{z}} .
\end{gathered}
$$

Thus $\tilde{E}_{z}$ and $\tilde{E}_{\perp}$ are obtained for every point on the path of the bead. Note that $\tilde{E}_{z}$ represents the longitudinal and $\tilde{E}_{\perp}$ the transverse component of the squared electrical field normalized to the power loss as given in Eq. (10).

\section{TEST SETUP}

In the test structure, the 36 copper cells are clamped together by truss rods. Its overall length is $1300 \mathrm{~mm}$. Cutoff pipes $100 \mathrm{~mm}$ in length and 20 and $40 \mathrm{~mm}$ in diameter, respectively, are attached to each end of the structure.

Proper alignment is assured by placing the structure on top of an optical bench. The field measurements are per-

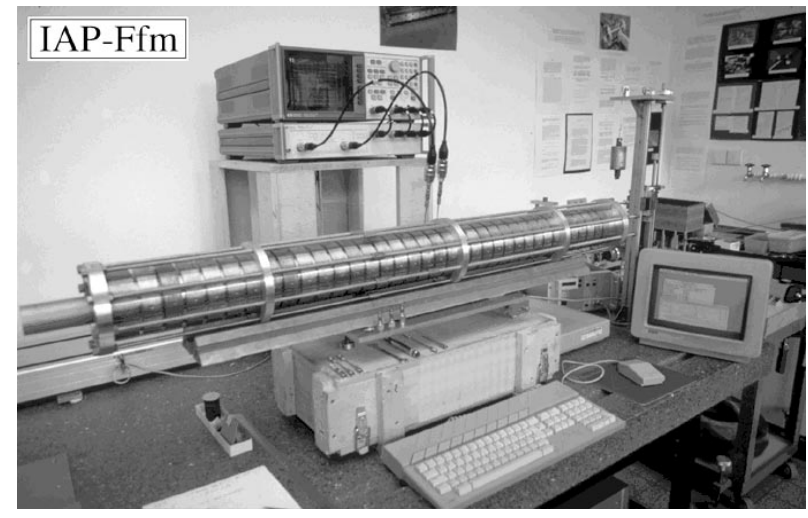

FIG. 5. Illustration of the test setup.

formed using a modified nonresonant bead pull technique $[11,12]$. Data are taken by a HP8753c network analyzer for 801 discrete positions along several paths parallel to the cavity axis. Figure 5 shows a picture of the test setup.

\section{RESULTS}

For the measurements, two ceramic beads with different shapes (needle, $\varnothing=0.6 \mathrm{~mm}$; length $=7.5 \mathrm{~mm}$; disk, $\varnothing=4.7 \mathrm{~mm}, 0.31 \mathrm{~mm}$ thick) were used. Both beads were calibrated in a $\mathrm{TM}_{010}$ pillbox for their longitudinal and transverse perturbation constants. The needle consisting of $\mathrm{Al}_{2} \mathrm{O}_{3}$ showed very stable values of $\hat{a}$ over the period of measurements whereas the disk revealed a significant change. It is believed that this was due to humidification of the bead surface. Its impact on the measurement results was, however, reduced by frequent recalibration of the beads.

In the following illustrations, two examples of trapped modes are shown: In Fig. 6 we show the longitudinal $E$ field of dipole mode \#15, and in Fig. 7 we show the

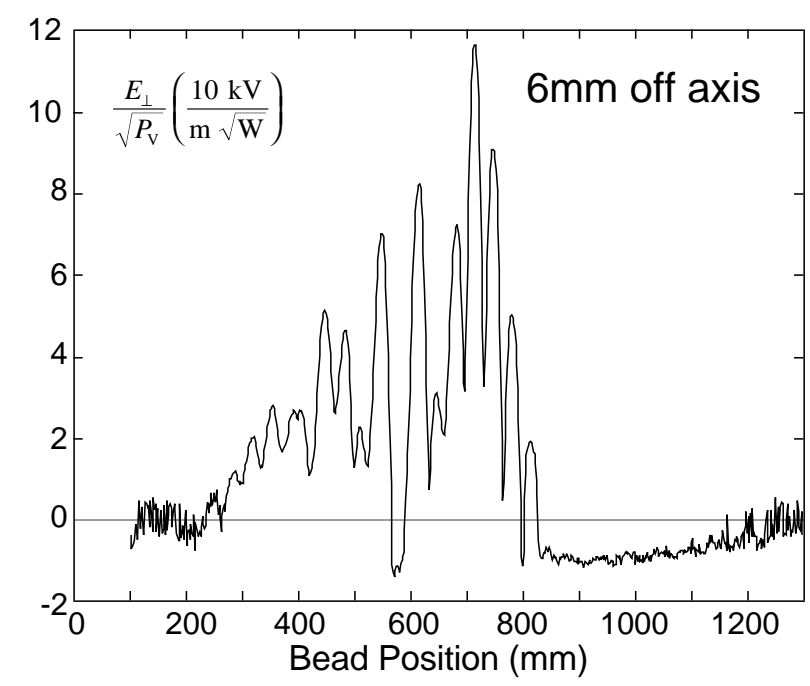

FIG. 6. Measurement: longitudinal $E$ field of mode \#15. 


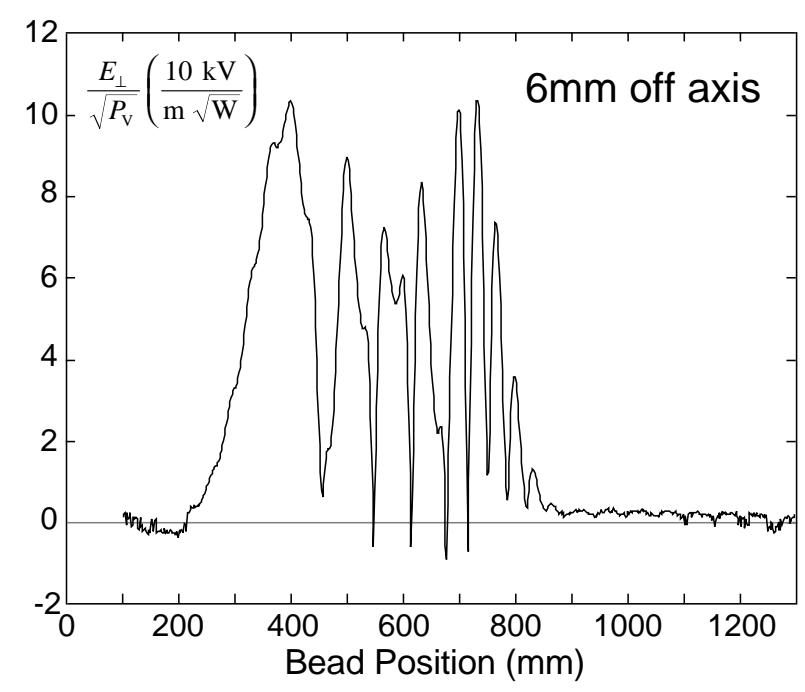

FIG. 7. Measurement: transverse $E$ field of mode \#15.

transverse $E$ field of the same mode. All fields were measured with a transverse offset (here $6 \mathrm{~mm}$ off axis), since for dipole modes the longitudinal electric field vanishes on axis.

Figures 8 and 9 show longitudinal and transverse electrical fields for mode \#16.

Comparing calculated and measured results, the field distributions showed very good agreement. On the average, resonant frequencies differ by less than $1 \%$, which is comparable to the accuracy of manufactured structures. The main parameters of modes \#15 and \#16 are summarized in Table I. The measured $Q$ 's are about $20 \%$ below the calculated values, which is acceptable for cups clamped together. While the transverse shunt impedance differs by about $8 \%$ for mode \#16-which is well inside the estimated $15 \%$ accuracy limit of the measurement-it shows a deviation of about twice as much for mode \#15.

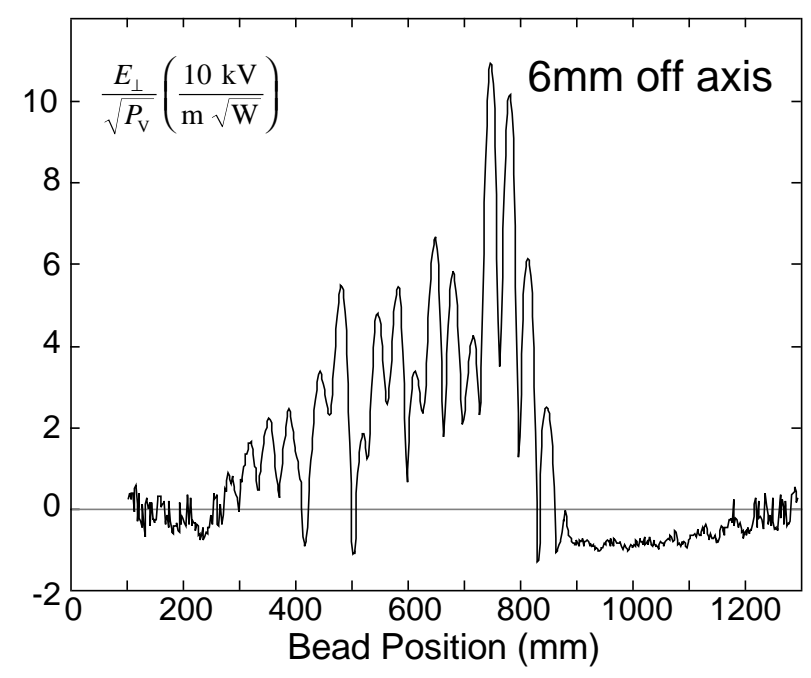

FIG. 8. Measurement: longitudinal $E$ field of mode \#16.

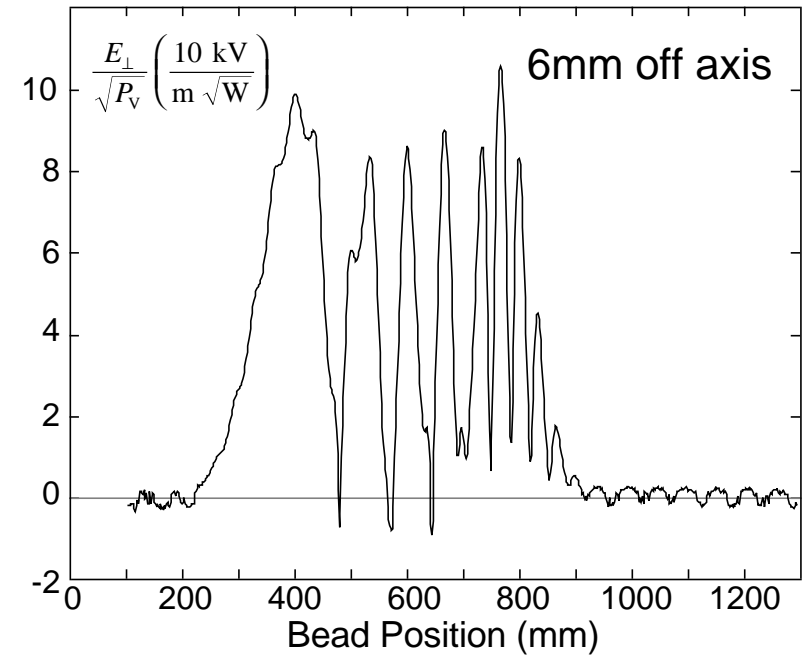

FIG. 9. Measurement: transverse $E$ field of mode \#16.

The values given for the transverse shunt impedance do not include transit time factors for electrons passing the cavity structures at the speed of light.

For the sake of comparison of measurements with the field distributions calculated by MAFIA and ORTHO, Fig. 10 shows the longitudinal $E$ field of mode \#15

Accordingly, Fig. 11 shows the transverse $E$ field of mode \#16. This result should be compared with Fig. 9 .

The normalized loss factor of the trapped dipole modes in the first dipole passband is nearly constant. For some modes the values calculated with MAFIA are shown in Table II. The deviation is of about $2.5 \%$. Only mode \#13 has a much lower value of about $866 \mathrm{~V} /\left(\mathrm{pC} \mathrm{m}^{2}\right)$. As measurements and computations show, this mode is only localized in the beam pipe and to a small extent in cell \#1. To suppress this mode, the diameter of the left beampipe was reduced afterwards from 40 to $20 \mathrm{~mm}$. The appearance in the first dipole passband is not significant: this mode is not determined by the cell geometry but, rather, only by the beampipe.

\section{STRUCTURE WITH DAMPER: TEST SETUP}

In order to measure the damped structure, a sheet of paper covered with graphite was inserted in cell \#18 (see Fig. 12). (This is only a model for test purposes

TABLE I. Transverse shunt impedance (transit time not regarded).

\begin{tabular}{lccc}
\hline \hline & & MAFIA & Measured \\
\hline Mode \#15 & $f(\mathrm{MHz})$ & 4170.99 & 4174.77 \\
& $R_{\perp} / Q(\Omega)$ & 521 & 626 \\
& $Q$ & 12379 & 9850 \\
Mode \#16 & $f(\mathrm{MHz})$ & 4185.57 & 4189.31 \\
& $R_{\perp} / Q(\Omega)$ & 556 & 605 \\
& $Q$ & 12471 & 10150 \\
\hline \hline
\end{tabular}




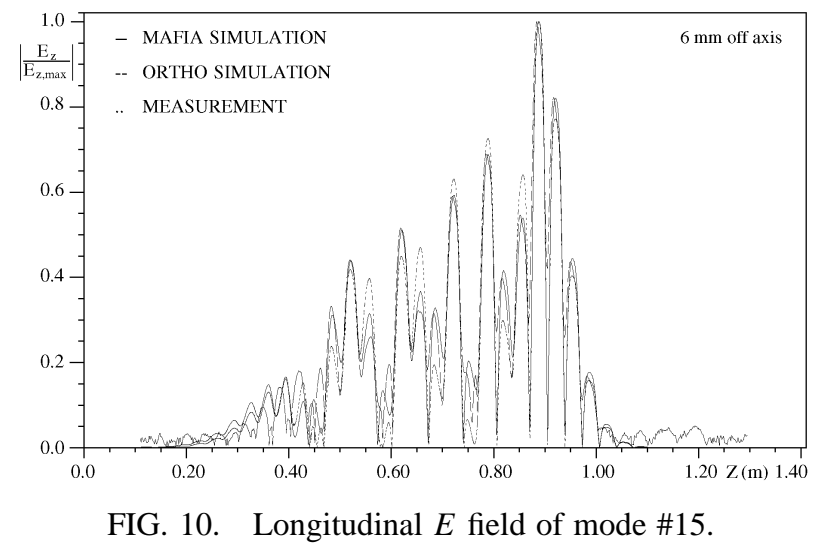

representing a damping unit like a walled slotted cell with four rectangular waveguides attached $[13,14]$.) The paper, with a thickness of $0.5 \mathrm{~mm}$, was laid against the inner wall of cell \#18 with the graphite facing the inside. The damping material could be inserted without demounting the structure: this avoided the influence of changed electrical contacts upon the measured $Q$ values after reassembly. This kind of damper does not disturb the rotational symmetry of the structure, thus again allowing for numerical simulations in a two-dimensional $r z$ plane. The field measurements were performed using again a modified nonresonant bead pull technique [15]. The time data were taken by a HP8720 network analyzer. The measurement setup was the same as described above (see Fig. 5).

\section{SIMULATION WITH DAMPING SHEET}

The computer simulation of the 36-cell structure with a very thin (about $1.5 \mu \mathrm{m}$ ) damping sheet in cell \#18 is scarcely possible with discretization methods since a sufficient accurate discretization would imply enormous storage demands. Therefore, a different procedure has been applied to yield quantitative theoretical results for

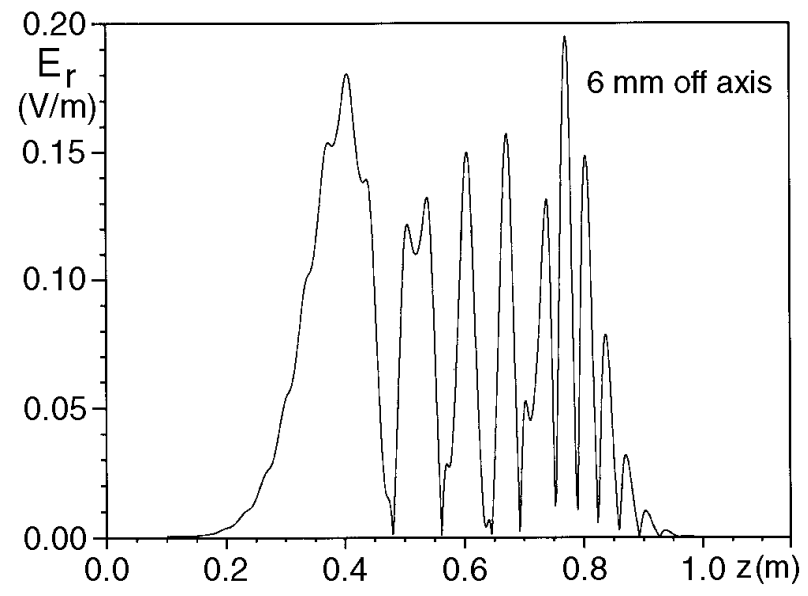

FIG. 11. MAFIA: transverse $E$ field of mode \#16.
TABLE II. Loss parameter of trapped modes.

\begin{tabular}{cccc}
\hline \hline Mode \# & $\begin{array}{c}f_{0, \mathrm{MAFIA}} \\
(\mathrm{MHz})\end{array}$ & $\begin{array}{c}f_{0, \text { measured }} \\
(\mathrm{MHz})\end{array}$ & $\begin{array}{c}\text { Loss factor } \\
{\left[\mathrm{V} /\left(\mathrm{pC} \mathrm{m}^{2}\right)\right]}\end{array}$ \\
\hline 15 & 4170.99 & 4174.77 & 4180 \\
16 & 4185.57 & 4189.31 & 4207 \\
19 & 4231.20 & 4230.57 & 4108 \\
20 & 4244.09 & 4243.90 & 4102 \\
21 & 4256.30 & 4256.31 & 4100 \\
22 & 4267.96 & 4268.27 & 4000 \\
\hline \hline
\end{tabular}

the damping effect. In case of small losses, the field distributions do not change considerably compared with the loss-free case. Thus it is reasonable to use the field distribution of the loss-free case to calculate the power loss by means of the perturbation theory. As the measurements suggest [16], the undisturbed field distribution may be used to calculate the damping effect for the 36-cell structure.

In practice, this was done with MAFIA in the following way: First, a loss-free field calculation was executed for the original 36-cell structure. In a second calculation, a thin sheet with a different material number was inserted in cell \#18, as shown in Fig. 13. The diagonally hatched area near the outer wall of cell \#18 is the graphite. Although a relatively coarse mesh would lead to a perfect approximation of the structure's shape, ${ }^{1}$ preceding studies have shown that smaller step sizes near all the edges resulted in much better agreement for the frequencies obtained in measurement, i.e., simulation, while the field distribution already agrees well enough to allow use of a coarse, quasiequidistant mesh. The frequency is very sensitive to an appropriate discretization and an appropriate bead [17].

During the solution of the eigenvalue problem, this material, as well as all walls of the structure, is treated as a perfect conductor. Afterwards, during postprocessing, all walls obtain the conductivity of copper, $\sigma=5.8 \times$ $10^{7} \mathrm{~S} / \mathrm{m}$, while the sheet in cell \#18 obtains a lower conductivity value.

At this point, some approximations are necessary because the sheet had to be made thicker than the foil actually used. The reasons are (i) that each material should be discretized with at least two steps in each coordinate

${ }_{1}$ i.e., vanishing geometric error

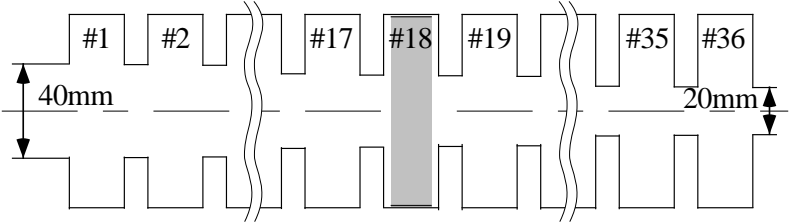

FIG. 12. 36-cell test structure with damper in cell \#18. 


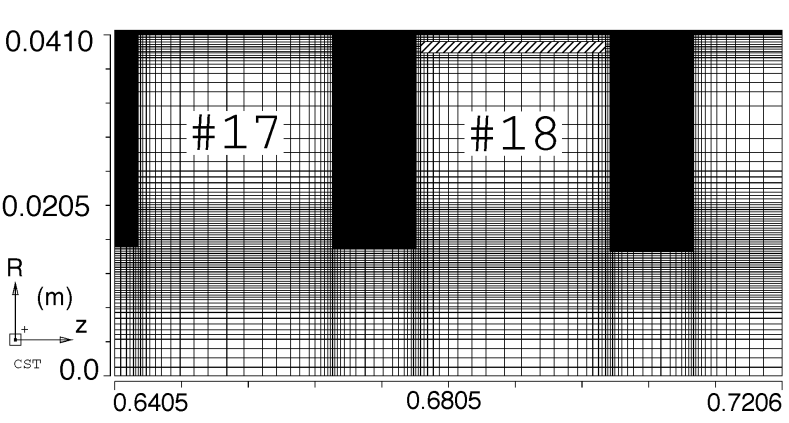

FIG. 13. Three cells out of the 36-cell structure. Cell \#18 with the damping material (diagonal hatching).

direction and (ii) that the quotient of largest to smallest step size should be kept with reasonable limits in order to limit the condition number of the matrix of the eigenvalue problem. Therefore, the sheet made of material representing the very thin graphite sheet was assigned a conductivity of $\sigma=5.0 \times 10^{6} \mathrm{~S} / \mathrm{m}$ instead of the conductivity of graphite $\sigma=1.25 \times 10^{5} \mathrm{~S} / \mathrm{m}$. In this way the resistivity is distributed over a greater volume with nearly the same damping effect.

After calculating the total stored energy $W_{\text {tot }}$ and the wall $\operatorname{losses}^{2} P_{\text {wall }}$, the quality factor $Q$ may be determined [18] for a mode with frequency $\omega$ by

$$
Q=\omega \frac{W_{\text {tot }}}{P_{\text {wall }}} .
$$

Finally, the frequency shift caused by the damping sheet in cell \#18 is obtained by expression (14) neglecting second order terms,

$$
\frac{\delta \omega}{\omega_{0}}=\frac{1}{2 Q}
$$

\section{MEASUREMENT METHOD WITH DAMPER}

The nonresonant bead pull technique was again applied. Here, only the longitudinal component of the electric field is of interest because it is sufficient to indicate the effect of the damper cell. Thus only a dielectric needle $\left(\mathrm{Al}_{2} \mathrm{O}_{3}\right.$, $\varepsilon_{r}=9.2,6 \mathrm{~mm}$ long and $1 \mathrm{~mm}$ in diameter) was used as the bead. A lumped circuit representation of the resonator combined with Slater's formula gives the electric field

$$
E^{2}=\frac{P_{\text {wall }}}{a_{z}} \frac{\left(1+k_{1}+k_{2}\right)^{2}}{2 \omega \sqrt{k_{1} k_{2}}}\left|\Delta s_{21}\right| .
$$

\section{RESULTS}

The bead was again calibrated in a $\mathrm{TM}_{010}$ pillbox to obtain the longitudinal perturbation constant $\left(a_{z}=9.34 \times\right.$

\footnotetext{
${ }^{2}$ by power loss method
}

$\left.10^{-20} \mathrm{~A} \mathrm{~s} \mathrm{~m}^{2} / \mathrm{V}\right)$. Since the measured modes were of dipole type the bead pull measurements were performed off axis with a distance of $7 \mathrm{~mm}$ from the axis of the structure. Several modes of the first dipole passband having significant field strength at the damper position (cell \#18) were chosen for the measurements since a considerable damping effect could be expected only in this case.

As described above, trapped modes in a detuned structure always change their phase advance from cell to cell from 0 to $\pi$ in total over all mode filled cells. To investigate the influence of the damper position, mode A with the $\pi$ end, mode $\mathrm{B}$ with the $\pi / 2$ part, and mode $\mathrm{C}$ with the 0 end at the damper position were chosen. In addition, all modes between $\mathrm{A}$ and $\mathrm{C}$ have been measured (see Fig. 14). The results are summarized in Table III.

First, the damping sheet was pressed to the inner wall of cell \#18 in order to obtain a symmetrical damper. This causes a relatively weak damping effect. The damping effect was enhanced by moving the sheet a few millimeters toward the iris, since the electrical field increases in this direction and the losses increase in proportion to conductivity and squared electrical field strength.

The simulation results are presented in the right-hand columns of Table III. It should be mentioned again that the $Q$ values computed are always higher than those derived by measurements. This is due to imperfect $\mathrm{rf}$ contacts between adjacent copper cells not taken into account in the simulation.

To compare the results from measurements and calculations, damping effects versus frequency are plotted for both in Fig. 14. As can be seen, the agreement is nearly perfect, except for the first two modes on the left-hand side. These two modes proved to be very sensitive to the position of the $\pi$-like end. Even a small disturbance moved the position of the $\pi$-like end to the next cell. Thus this discrepancy was to be expected.

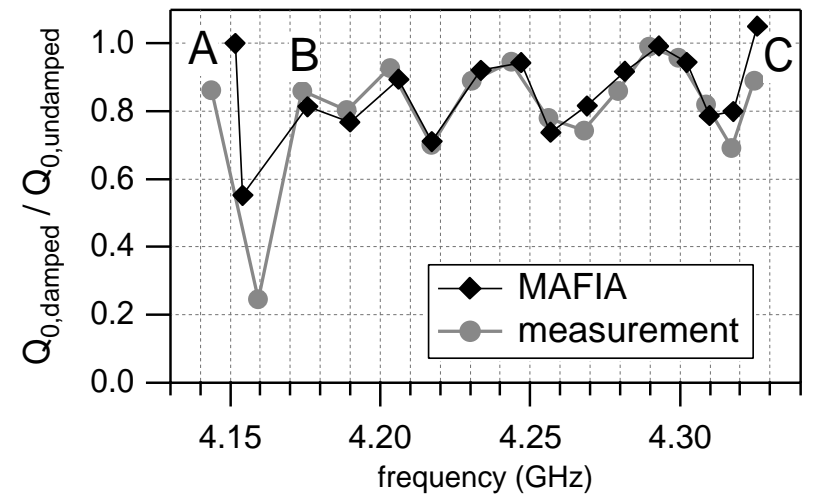

FIG. 14. Comparison of calculated and measured damping effect (reduction of $Q_{0}$ ). 
TABLE III. Measurement and MAFIA results.

\begin{tabular}{ccccc}
\hline \hline & \multicolumn{2}{c}{ Measurement } & \multicolumn{2}{c}{ MAFIA } \\
$f_{0}(\mathrm{MHz})$ & $Q_{0}$ & $Q / Q_{0}$ & $Q_{0}$ & $Q / Q_{0}$ \\
\hline 4143.830 & 10353 & 0.862 & 13650 & 1.000 \\
4159.260 & 10264 & 0.244 & 12552 & 0.553 \\
4173.972 & 10464 & 0.860 & 12641 & 0.814 \\
4188.848 & 10157 & 0.803 & 12736 & 0.768 \\
4203.234 & 10193 & 0.928 & 12839 & 0.894 \\
4217.087 & 11291 & 0.701 & 12947 & 0.711 \\
4230.494 & 10848 & 0.889 & 13066 & 0.921 \\
4243.603 & 10997 & 0.945 & 13185 & 0.942 \\
4256.029 & 10490 & 0.780 & 13308 & 0.737 \\
4267.888 & 11174 & 0.744 & 13436 & 0.817 \\
4279.207 & 11185 & 0.860 & 13570 & 0.917 \\
4289.655 & 11447 & 0.991 & 13699 & 0.992 \\
4299.424 & 11396 & 0.958 & 13833 & 0.944 \\
4308.621 & 11757 & 0.820 & 13962 & 0.787 \\
4317.108 & 11918 & 0.692 & 14094 & 0.800 \\
4324.609 & 12112 & 0.889 & 14229 & 1.051 \\
\hline \hline
\end{tabular}

\section{CONCLUSION}

With the 36-cell structure, the existence of trapped modes in a detuned accelerating structure could be proven by means of numerical calculation as well as by measurement. In this fashion, the reliability of ORTHO was demonstrated by MAFIA calculations and measurements. Thus it seems possible to make reliable predictions on the SBLC structure with 180 cells. The behavior of a single damper cell in a detuned structure meets expectations: the damping effect is proportional to the field amplitude in the damper cell. But, going from former measurements, a strong influence of mode geometry on damping has been anticipated [19]. However, weak as well as strong damping did not change the mode geometry significantly. The damping effect is independent of phase advance.

The simulation of a damped structure is not an easy task; however, the results from MAFIA show a good conformity with measurement results. Thus one may conclude that the numerical representation of the damper was successful.

Detuning suppresses synchronous dipole modes with an extremely high loss factor. Comparatively, nearly all modes of the first dipole band in a detuned structure have significant loss factors: the $\pi$-like end of all these modes can be coupled very well to the particle beam. Thus one has to pay attention to a much higher quantity of modes; their shunt impedances, however, are lower. A single HOM coupler cell will not be able to cause sufficient damping because there is no cell occupied by all these trapped modes. A possible completion is intrinsic HOM dampers on the iris of some or all cells as described in [3].

\section{ACKNOWLEDGMENTS}

This work was supported by DESY/Hamburg and BMBF under Contract No. 06OF841.

[1] P. Hülsmann, Ph.D. thesis, Institut für Angewandte Physik, Frankfurt am Main, 1992.

[2] U. van Rienen, Part. Accel. 41, 173 (1993).

[3] M. Dohlus, H. Hartwig, N. Holtkamp, S. Ivanov, V. Kaljuzhny, and A. Naboka, DESY Report No. 96-169, 1996.

[4] N. Holtkamp, in Proceedings of the Particle Accelerator Conference and International Conference on High-Energy Accelerators, Vancouver, Canada, 1997 (IEEE, Piscataway, NJ, 1998).

[5] T. Weiland, AEÜ 31, 116 (1977).

[6] U. van Rienen and T. Weiland, Part. Accel. 20, 239 (1986).

[7] T. Weiland, Part. Accel. 17, 227 (1985); DESY Report No. 84-111, 1984.

[8] T. Weiland and B. Zotter, Part. Accel. 11, 143 (1981).

[9] B. Krietenstein, O. Podebrad, U. van Rienen, T. Weiland, H.-W. Glock, P. Hülsmann, H. Klein, M. Kurz, and C. Peschke, in Proceedings of the Particle Accelerator Conference and International Conference on High-Energy Accelerators, Dallas, Texas, 1995 (IEEE, Piscataway, NJ, 1996).

[10] L. C. Maier and J.C. Slater, J. Appl. Phys. 23(1), 68 (1952).

[11] C. W. Steele, IEEE Trans. Microwave Theory Tech. 14(2), 70 (1966).

[12] M. Kurz, Ph.D. thesis, Institut für Angewandte, Frankfurt am Main, 1993.

[13] W.F. O. Müller, P. Hülsmann, M. Kurz, H.-W. Glock, and H. Klein, in Proceedings of the XVII International Linear Accelerator Conference, Tsukuba, Japan, 1994 (KEK, Iburaki Japan, 1994).

[14] R. Brinkmann et al., TESLA Collaboration and SBLC Collaboration, DESY/Hamburg Report No. DESY-97048::V.1, Fig. 4.2.15, 1997, p. 1183.

[15] P. Hülsmann, M. Kurz, and H. Klein, Electron. Lett. 27, 1727 (1991).

[16] W.F. O. Müller, P. Hülsmann, M. Kurz, C. Peschke, H. Klein, U. van Rienen, T. Weiland, and M. Dohlus, in Proceedings of the XVIII International Linear Accelerator Conference, Geneva, Switzerland, 1996 (CERN, Geneva, 1996), p. 713.

[17] M. Kurz, W. F. O. Müller, U. Niermann, P. Hülsmann, H. Klein, U. van Rienen, O. Podebrad, T. Weiland, and M. Dohlus, in Proceedings of the 5th European Particle Accelerator Conference, Barcelona, Spain, 1996 (Institute of Physics, Bristol, UK, 1996).

[18] T. Weiland, Part. Accel. 56, 61 (1996).

[19] M. Dohlus, M. Marx, N. Holtkamp, P. Hülsmann, W. F. O. Müller, M. Kurz, H.-W. Glock, and H. Klein, in Proceedings of the Particle Accelerator Conference and International Conference on High-Energy Accelerators, Dallas, Texas, 1995 (IEEE, Piscataway, NJ, 1996). 